\title{
The gluon propagator from large asymmetric lattices
}

\section{Orlando Oliveira*}

Department of Physics, University of Coimbra, 3004516 Coimbra, Portugal

E-mail: orlando@teor.fis.uc.pt

\section{P. J. Silva}

Department of Physics, University of Coimbra, 3004516 Coimbra, Portugal E-mail: psilva@teor.fis.uc.pt

\section{E.-M. llgenfritz}

Humboldt-Universität zu Berlin, Institut für Physik, Newtonstr. 15, 12489 Berlin, Germany, E-mail: ilgenfri@physik.hu-berlin.de

\section{A. Sternbeck}

CSSM, School of Chemistry \& Physics, The University of Adelaide, SA 5005, Australia

E-mail: andre.sternbeck@adelaide.edu.au

The Landau-gauge gluon propagator is computed for the $S U(3)$ gauge theory on lattices up to a size of $32^{3} \times 200$. We use the standard Wilson action at $\beta=6.0$ and compare our results with previous computations using large asymmetric and symmetric lattices. In particular, we focus on the impact of the lattice geometry and momentum cuts to achieve compatibility between data from symmetric and asymmetric lattices for a large range of momenta.

The XXV International Symposium on Lattice Field Theory

July 30 - August 4, 2007

Regensburg, Germany

\footnotetext{
* Speaker.
} 


\section{Introduction and motivation}

The investigation of the Landau-gauge gluon propagator

$$
D_{\mu v}^{a b}(q)=\delta^{a b}\left(\delta_{\mu v}-\frac{q_{\mu} q_{v}}{q^{2}}\right) D\left(q^{2}\right)
$$

in lattice QCD dates back to more than 20 years. Despite the efforts made by a number of authors, there are questions which remain to be answered. For large momenta, let us say $q>2 \mathrm{GeV}$, the results from different groups provide a consistent picture of the propagator and agree well with calculations performed using other non-perturbative techniques. On the other hand, the infrared limit is still an open issue and has been a field of intense research in the last years (see the contributions to this conference, e.g. [1], and references therein). From the point of view of lattice simulations, the questions to be answered yet are numerous and sometimes are even not easy to handle with. For example, it is still under debate how to deal with the Gribov ambiguity in lattice simulations, and how close present lattice data come to Landau-gauge gluodynamics in the continuum and infinite-volume limit.

In order to access the infrared limit of the gluon propagator, two of us (O.O. and P.J.S.) recently proposed and explored the use of large asymmetric lattices [2, 3, 4], i.e. $L^{3} \times T$ with $T \gg L$. The price of relying on such kind of lattices are the control, or the lack of it, of additional finitevolume effects coming from a breaking of the $Z_{4}$ symmetry, a remnant of the $O(4)$ continuum symmetry on a symmetric hypercubic lattice. When previous studies on symmetric lattices have shown strong finite-volume effects in the infrared region [5], the situation is more dramatic for asymmetric lattices. For example, there the gluon dressing function $Z\left(q^{2}\right)=q^{2} D\left(q^{2}\right)$ computed at equal time-like and spatial momenta are not necessarily compatible within pure statistical errors in the low-momentum region.

On the other hand, the access to very low momenta is much more computationally intensive in simulations on symmetric lattices compared to those on asymmetric ones. Therefore, if somehow the asymmetry-induced finite-volume effects were brought under control, data at much lower momenta than currently available could be obtained. Indeed, then still the infinite volume limit has to be taken, but in a situation where more data were available in the infrared momentum region.

Having now access to a considerably larger spatial volume for the asymmetric case, in this study we report on some first results obtained comparing data on symmetric and asymmetric lattices, namely $16^{3} \times 256,18^{3} \times 256,32^{3} \times 200$ and $32^{4}$. In particular, we look for regions in the lattice momentum space where the differences between time-like and spatial momenta disappear and where not. For our simulations we use the standard Wilson gauge action with $\beta=6.0$ fixed. This value corresponds to an inverse lattice spacing of about $a^{-1}=1.94 \mathrm{GeV}$. To relate our data at the different lattice momenta to their continuum counterparts we use

$$
q_{\mu}=\frac{2}{a} \sin \left(\frac{\pi n_{\mu}}{L_{\mu}}\right), \quad n_{\mu}=0,1, \ldots, L_{\mu}-1,
$$

where $L_{\mu}$ is the lattice extent in direction $\mu$. Definitions and details on the gauge fixing are given in [7] and for the $32^{3} \times 200$ data in [8]. In the following, whenever possible, a $Z_{3}$ average over equivalent momenta is performed. 

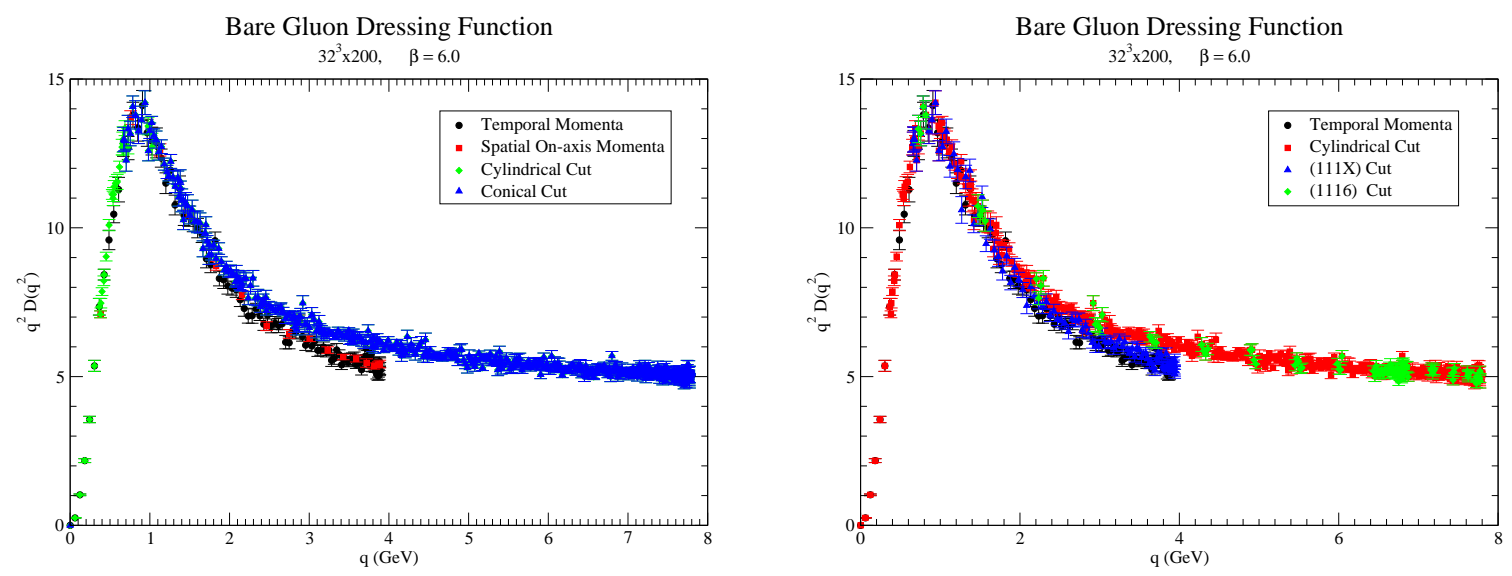

Figure 1: The gluon dressing function on a $32^{3} \times 200$ lattice. The different momentum cuts are discussed in the text.

2. Asymmetric lattice: $32^{3} \times 200$

The gluon propagator and dressing function were computed for 39 configurations using a $32^{3} \times 200$ lattice. The data shows discretization effects similar to those seen in the symmetric lattices. Indeed, it is well-known that for momenta above $q>1 \mathrm{GeV}$ the propagator is not a simple function of $q^{2}=q_{\mu}^{2}$ alone. The traditional approach is to apply momenta cuts [6] which reduce the dependence of the propagator and the dressing function on other $Z_{4}$ (in our case $Z_{3}$ ) invariants to a unique curve. This is better seen in the gluon dressing function. In order to illustrate this effect, in Fig. 1 we plot the gluon dressing function for different choices of momenta (purely time-like and different cuts of spatial momenta).

The plot shows that, within our limited statistics, there is very good agreement between the dressing functions computed using purely spatial on-axis and purely temporal momenta. The figure does not include the dressing function for all purely spatial momenta. However, in what concerns the purely spatial momenta, the gluon dressing function for on-axis momenta evolves typically along the lower edge of the spatial (including off-axis) momentum data. The diagonal choice of momenta, i.e. the cylindrical cut [6] where $n_{\mu} \approx \pm n_{v}$ (see the left plot in Fig. 1) picks up an unique propagator which is slightly above the propagator for the on-axis choice for momenta at $q$ larger than $\sim 1 \mathrm{GeV}$. Note that this "democratic" choice of momenta has been successfully used to suppress discretization effects such that data from different volumes and lattice spacings match at larger momenta.

In the right plot of Fig. 1 we also show two naive generalizations to the asymmetric case of the on-axis case and of a "democratic" choice of momenta (the cylinder cut), labelled as $(111 X)$ and (1116) cuts. The former includes momenta only of type $n_{\mu}=\left( \pm 1, \pm 1, \pm 1, \pm n_{t}\right)$ with $n_{t}=$ $0,1, \ldots, T / 2$ (softening of on-axis momenta), while the latter includes momenta defined around the direction $n_{\mu}=\left( \pm n, \pm n, \pm n, \pm 6 n_{t}\right)$ with $n=0,1, \ldots, L-1$ and $n_{t}=n, n \pm 1, n \pm 2$. This direction is close to the diagonal in the elongated volume (remember that $T / L=6.25$ ). Note that the $(111 X)$ cut reproduces the results of the symmetric lattice for the on-axis choice of momenta, while the (1116) cut follows the symmetric lattice data for the cylindrical and conical cuts. Given that the cylindrical and conical cuts seem to reduce the finite-volume effects for momenta above $1 \mathrm{GeV}$, 

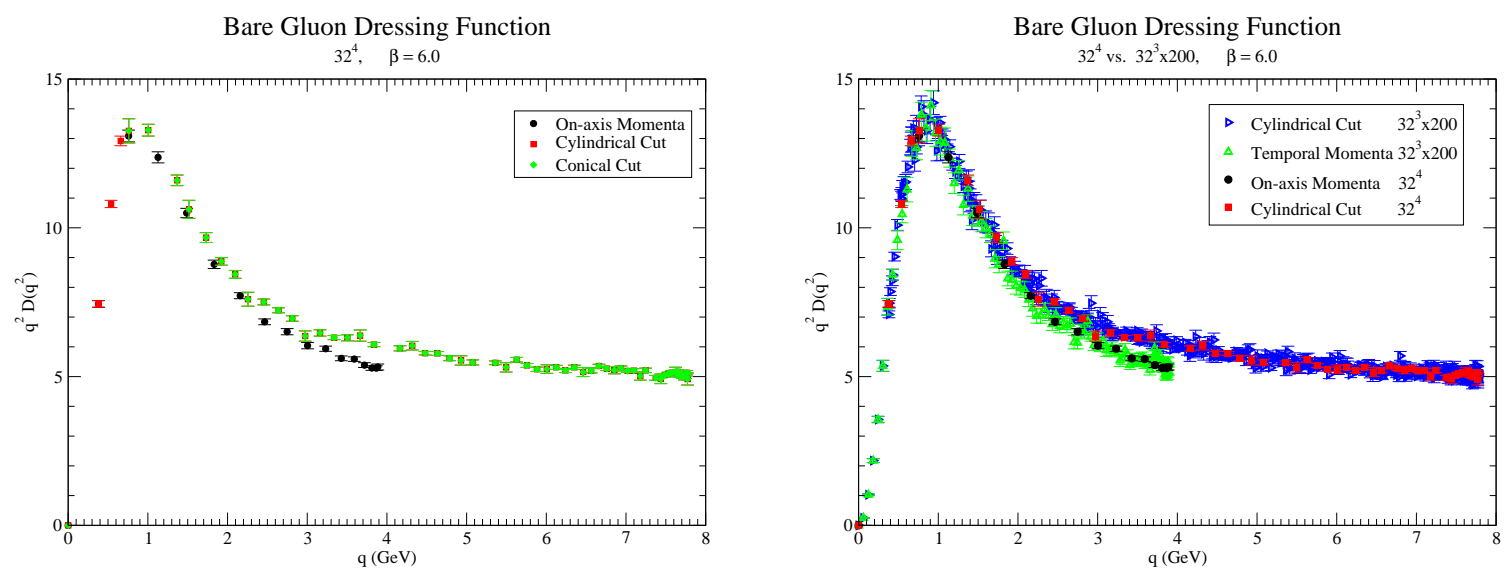

Figure 2: The gluon dressing function for a $32^{4}$ lattice is shown on the left hand side. The right figure compares the same data with those on a $32^{3} \times 200$ lattice. Different cuts have been applied to the data.

Fig. 1 suggests that for the asymmetric lattices one should use the (1116) cut, or a variant of it, for that momentum region.

\section{Symmetric lattice: $32^{4}$}

The gluon dressing function $Z\left(q^{2}\right)=q^{2} D\left(q^{2}\right)$, computed for an ensemble of 50 gauge configurations on a $32^{4}$ lattice, is given in the left plot of Fig. 2 applying various cuts. The lattice data shows similar discretization effects as for the asymmetric lattice. This is illustrated in the right plot of the same figure. There, data for the two lattices $32^{4}$ and $32^{3} \times 200$ are shown for two different momentum cuts at larger momenta and good agreement is found. Moreover, within our limited statistics, the cuts produce similar effects for both lattices where the on-axis data lie in both cases systematically below cylinder-cut data for $q>2 \mathrm{GeV}$.

In Fig. 3 the lattice gluon dressing function for a $32^{3} \times 200$ and a $32^{4}$ lattice are compared for momenta below $2 \mathrm{GeV}$. Again, two different cuts (on-axis and cylindrical) are considered. We find that in this momentum range the dressing functions $Z\left(q^{2}\right)$ for the two lattice geometries are in good agreement, even though we cannot compare at the low-lying momenta. A comparison with data from larger symmetric lattices is necessary to become more confident in this. At least, the good matching between $0.5 \mathrm{GeV}$ and $2 \mathrm{GeV}$ is encouraging in what concerns the use of asymmetric lattices to extract reliable infrared properties in future lattice simulations.

\section{The impact of the spatial volume}

Now we discuss the volume dependence of the propagator $D(q \neq 0)$ as function of the physical momentum $q$. In Fig. 4 we compare the gluon propagator on one hand and the dressing function on the other hand for various asymmetric lattices, for $32^{4}$ (all at $\beta=6.0$ ) and for the continuum DysonSchwinger solution of Ref. [10]. All propagators were renormalized according to the condition

$$
\left.D\left(q^{2}\right)\right|_{q^{2}=\mu^{2}}=\frac{1}{\mu^{2}}
$$




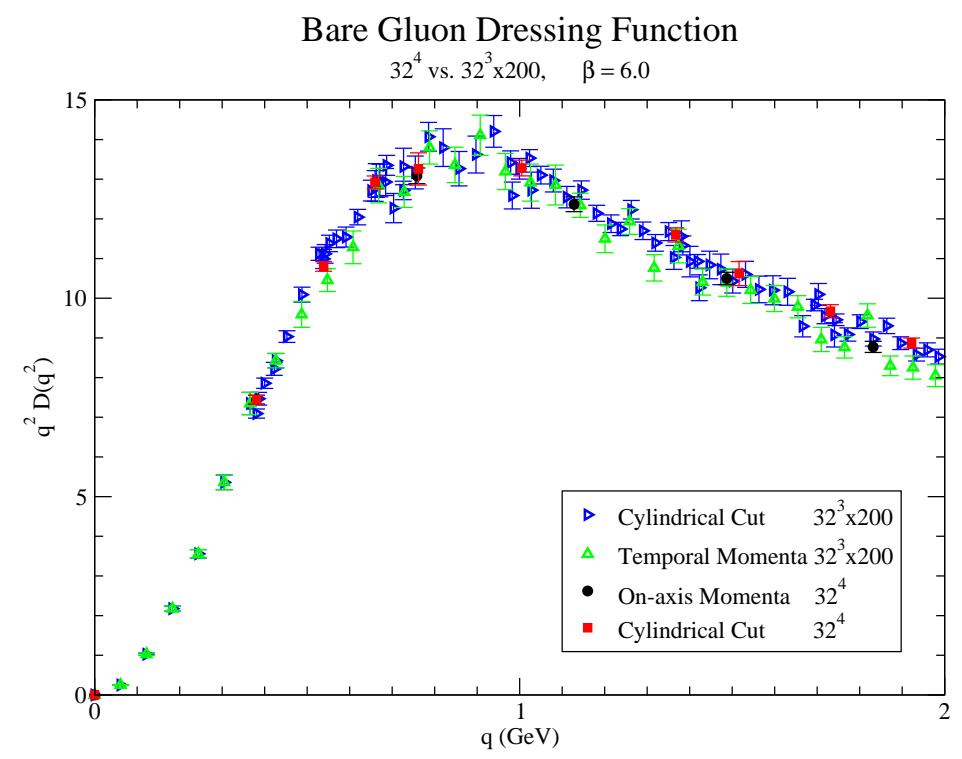

Figure 3: The gluon dressing function at low momenta for the $32^{4}$ and the $32^{3} \times 200$ lattices for two different momenta cuts.
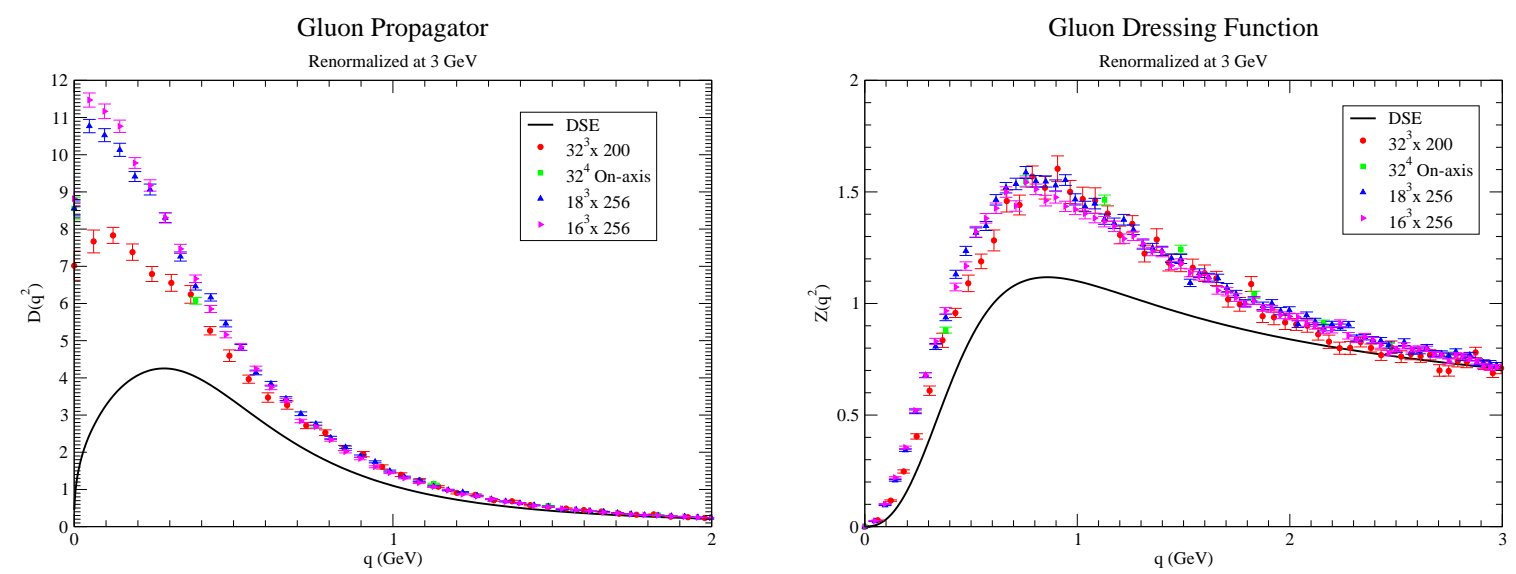

Figure 4: The gluon propagator (left) and the gluon dressing function (right) for various asymmetric lattices and for $32^{4}$ at $\beta=6.0$. compared with the DSE solution.

with the choice $\mu=3 \mathrm{GeV}$. For asymmetric lattices, the data is for time-like (on-axis) momenta. For the symmetric lattice, the plot includes only on-axis momenta. Fig. 4 shows that the two results become closer as the lattice volume increases.

\section{Fitting the IR gluon dressing function}

In previous investigations it was verified that the lattice gluon dressing function and the continuum Dyson-Schwinger solution are well described not by a pure power law but by both functions

$$
Z_{I}\left(q^{2}\right)=\omega\left(\frac{q^{2}}{q^{2}+\Lambda^{2}}\right)^{2 \kappa}, \quad Z_{I I}\left(q^{2}\right)=\omega \frac{\left(q^{2}\right)^{2 \kappa}}{\left(q^{2}\right)^{2 \kappa}+\left(\Lambda^{2}\right)^{2 \kappa}}
$$




\begin{tabular}{rlllll}
\hline Lattice & $q_{\max }$ & $\kappa$ & $\Lambda$ & $\chi^{2} /$ d.o.f. & \# conf \\
\hline $16^{3} \times 256$ & 664 & $0.5090_{-20}^{+19}$ & $409_{-4}^{+4}$ & 0.71 & 155 \\
& & & & & \\
$18^{3} \times 256$ & 711 & $0.5320_{-30}^{+28}$ & $389_{-6}^{+5}$ & 1.14 & 150 \\
& & & & & \\
$32^{3} \times 200$ & 728 & $0.532_{-12}^{+12}$ & $465_{-23}^{+25}$ & 1.28 & 39 \\
\hline
\end{tabular}

Table 1: Parameters corresponding to a fit of the lattice gluon dressing function according to $Z_{I}\left(q^{2}\right)$. $q_{\max }$ and $\Lambda$ are given in $\mathrm{MeV}$.

\begin{tabular}{rlllll}
\hline Lattice & $q_{\max }$ & $\kappa$ & $\Lambda$ & $\chi^{2} /$ d.o.f. & \# conf \\
\hline $16^{3} \times 256$ & 664 & $0.5077_{-17}^{+16}$ & $409_{-3}^{+4}$ & 0.69 & 155 \\
& & & & & \\
$18^{3} \times 256$ & 711 & $0.5266_{-21}^{+29}$ & $391_{-7}^{+3}$ & 1.09 & 150 \\
& & & & & \\
$32^{3} \times 200$ & 728 & $0.528_{-8}^{+10}$ & $464_{-23}^{+22}$ & 1.16 & 39 \\
\hline
\end{tabular}

Table 2: Parameters corresponding to a fit of the lattice gluon dressing function according to $Z_{I I}\left(q^{2}\right) . q_{\max }$ and $\Lambda$ are given in $\mathrm{MeV}$.

for momenta below $\sim 700 \mathrm{MeV}$.

The results of fitting the lattice dressing function for purely temporal momenta with $Z_{I}$ and $Z_{I I}$ are reported in tables 1 and 2, respectively; $q_{\max }$ is the highest momentum included in the fits. Note that, the exponent $\kappa$ agrees within one standard deviation for the two largest volumes. Furthermore, for these lattices, $\kappa$ agrees with the estimate of O.O. and P.J.S. [3], $\kappa \sim 0.53$, from using ratios of propagators to suppress the volume dependence. The ratio method discussed in [3], if applied to the $32^{3} \times 200$ data, estimates $\kappa=0.565 \pm 0.040$ for the infrared exponent.

The results give $\kappa$ consistently above 0.5 . If this really represents the infrared asymptotics, it supports a vanishing $q \rightarrow 0$ limit of the gluon propagator $D(q \neq 0)$. Moreover, one should keep in mind that the fits to a pure power law provide always a $\kappa<0.5$, with $\kappa$ increasing with the lattice volume. It should be noted, however, that so far lattice simulations have always reported a finite and not vanishing gluon propagator at zero momentum [12].

\section{Results and Conclusions}

In this work the gluon propagator and dressing function has been analysed for various asymmetric lattices and a comparison to $32^{4}$ data has been done. Despite the observed finite-volume effects, for volumes as large as $32^{3} \times 200$ the dressing function $Z\left(q^{2}\right)$ for purely temporal momenta agrees well, within the available statistics, with the corresponding function for purely spatial on-axis momenta.

In what concerns the $32^{3} \times 200$ and $32^{4}$ data, the momentum cuts produce similar results for the full range of momenta, i.e. the gluon propagator/dressing function for on-axis momenta are systematically below cone-cut or cylinder-cut data for $q>1 \mathrm{GeV}$. 
The behaviour of the lattice infrared gluon dressing function $Z\left(q^{2}\right)$ is well described by the two ansätze $Z_{I}$ and $Z_{I I}$ for $q<700 \mathrm{MeV}$. The fits to the data provide $\kappa$ values which support a vanishing zero momentum limit of the gluon propagator for all the lattices reported here. The measurement of the infrared exponent $\kappa$ for the two larger lattices suggests a value $\kappa \sim 0.53$, in agreement with the estimate discussed in [3].

\section{Acknowlegdements}

P.J.S. acknowledges F.C.T. financial support via grant SFRH/BD/10740/2002. This work was supported in part by F.C.T. under contracts POCI/FP/63436/2005 and POCI/FP/63923/2005. E.M. I. thanks the DFG for support through the Forschergruppe FOR 465. A. S. is supported by the Australian Research Council.

\section{References}

[1] C. S. Fischer et al., arXiv:0709.3205 [hep-lat] (2007); A. Sternbeck, et al., PoS(LATTICE2007)340, (2007); I. L. Bogolubsky et al., PoS(LATTICE2007)290, (2007); PoS(LATTICE2007)318; O. Oliveira, et al., PoS(LATTICE2007)332, (2007); A. Cucchieri, et al, PoS(LATTICE2007)297, (2007).

[2] O. Oliveira, P. J. Silva, Braz. J. Phys. 37, 201 (2007).

[3] O. Oliveira, P. J. Silva, arXiv:0705.0964[hep-lat] (2007).

[4] O. Oliveira, P. J. Silva, AIP Conf. Proc. 756, 290 (2005).

[5] See, for example, A. Cucchieri, T. Mendes, O. Oliveira, P. J. Silva, arXiv:0705.3367[hep-lat] (2007).

[6] D. B. Leinweber, J. I. Skullerud, A. G. Williams, C. Parrinello, Phys. Rev. D58 (1998) 031501; Phys. Rev. D60 (1999) 094507.

[7] P. J. Silva, O. Oliveira, Phys. Rev. D74, 034513 (2006).

[8] A. Sternbeck, E.-M. Ilgenfritz, M. Müller-Preussker, A. Schiller, Phys. Rev. D72, 014507 (2005).

[9] O. Oliveira, P. J. Silva, PoS(LAT2005)287, (2005).

[10] C. S. Fischer, M. R. Pennington, Phys. Rev. D73, 034029 (2006).

[11] O. Oliveira, P. J. Silva, EPJA31, 790 (2007)

[12] See, for example, E.-M. Ilgenfritz, M. Müller-Preussker, A. Sternbeck, A. Schiller, I. L. Bogolubsky, Braz. J. Phys. 37, 193 (2007). 